\title{
Tracking of nutrient intakes in adolescence: the experiences of the Young Hearts Project, Northern Ireland
}

\author{
P. J. Robson ${ }^{1 *}$, A. M. Gallagher ${ }^{1}$, M. B. E. Livingstone ${ }^{1}$, G. W. Cran ${ }^{2}$, J. J. Strain ${ }^{1}$, J. M. Savage ${ }^{3}$ and \\ C. A. G. Boreham ${ }^{4}$ \\ ${ }^{1}$ Northern Ireland Centre for Diet and Health (NICHE), University of Ulster, Coleraine BT52 1SA, Northern Ireland, UK \\ ${ }^{2}$ Department of Epidemiology and Public Health, The Queen's University of Belfast, Institute of Clinical Science, Belfast \\ BT12 6BJ, Northern Ireland, UK \\ ${ }^{3}$ The Department of Child Health, The Queen's University of Belfast, Clinical Institute, Royal Victoria Hospital, Belfast BT12 \\ 6BJ, Northern Ireland, UK \\ ${ }^{4}$ School of Leisure and Tourism, University of Ulster, Jordanstown BT37 OQB, Northern Ireland, UK
}

(Received 7 July 1999 - Revised 16 December 1999 - Accepted 6 March 2000)

\begin{abstract}
This study evaluated the tracking of energy and nutrient intakes, assessed by diet history, in a random sample of adolescents (boys $n$ 225, girls $n$ 230) at baseline (age 12 years), and subsequently at age 15 years. Median energy $(\mathrm{MJ} / \mathrm{d})$ and macronutrient $(\mathrm{g} / \mathrm{d})$ intakes increased significantly (all $P<0.001$ ) with increasing age in the boys. The girls' reported energy intake $(\mathrm{MJ} / \mathrm{d})$ remained stable over time, despite significant increases in BMI, weight and \% body fat. Age-related changes in the girls' macronutrient intakes were inconsistent. When expressed in terms of nutrient density, the diets of both sexes became significantly richer, over time, in total folate (both sexes, $P<0.01$ ), but poorer in Ca (boys $P<0.01$, girls $P<0.001$ ) and riboflavin (both sexes $P<0.001)$. Vitamin $\mathrm{B}_{6}(P<0.001)$ and $\mathrm{Fe}(P<0.05)$ densities increased in the boys, while the thiamin density of the girls' diets decreased $(P<0.001)$. Tracking, defined as maintenance of rank over time, was summarised using weighted kappa statistics $(\kappa)$. There were some significant changes in intakes at the group level; however, tracking of energy and nutrients in both sexes was only poor to fair $(\kappa<0.40)$, indicating substantial drift of individuals between classes of intake over time. Particularly poor tracking was evident for $\%$ energy from sugars $(\kappa$ 0.09 ) and total fat (к 0.09) in the girls' diets. In conclusion, the poor to fair tracking observed in this cohort suggests that individual dietary patterns exhibited at 12 years of age are unlikely to be predictive of energy and nutrient intake at age 15 years.
\end{abstract}

Adolescents: Nutrient density: Nutrient intake tracking: Northern Ireland

It is widely accepted that physiological risk factors for IHD, such as unfavourable lipid and blood pressure profiles, are likely to 'track' from early life into adulthood (Lauer et al. 1988; Webber et al. 1991; Porkka et al. 1994; Raitakari et al. 1994; Twisk et al. 1997b). Tracking has been defined as the maintenance of relative position in rank of behaviour over time, such that subjects who rank highly for unfavourable risk profiles at a young age are likely to maintain their ranks through into adulthood (Kelder et al. 1994; Twisk et al. 1997a).

The evidence that dietary patterns formed in early life may track into adulthood, and thus influence the progression of chronic disease, has been cited as the rationale for targeting 'healthy eating' programmes at children and adolescents (Kelder et al. 1994; Boulton et al. 1995; Singer et al. 1995). While moderate tracking of selected nutrients has been observed in younger children (Stein et al. 1991; Boulton et al. 1995; Singer et al. 1995), there is limited and inconsistent evidence for similar tracking in adolescents (Boulton et al. 1995; Welten et al. 1997). The majority of studies concerning nutrient intakes in older children have been cross-sectional (Adamson et al. 1992; Crawley, 1993; Anderson et al. 1994; Strain et al. 1994; Samuelson et al. 1996; Hurson \& Corish, 1997).

Consequently, the aim of the present study was to use longitudinal data from the Young Hearts Study, conducted

\footnotetext{
Abbreviations: EAR, estimated average requirement; EI, energy intake; $\kappa$, weighted kappa value.

* Corresponding author: Dr Paula Robson, fax +44 1265 324965, email p.robson@ulst.ac.uk
} 
in Northern Ireland, UK, to evaluate the extent of tracking of energy and nutrient intakes reported at baseline by a random sample of adolescents aged 12 years, and 3 years later at follow-up.

\section{Methodology \\ Subjects}

The Young Hearts Project is an ongoing longitudinal study of the prevalence of risk factors for IHD in a random sample of subjects from Northern Ireland. The baseline survey of 1015 randomly-selected schoolchildren (251 12year-old boys, 258 12-year-old girls, 25215 -year-old boys and 254 15-year-old girls) was completed in 1990 with a response rate of $78 \%$. The resulting cohort accounted for a $2 \%$ sample of each of the two respective age populations in Northern Ireland. Sampling procedures are described fully elsewhere (Boreham et al. 1993). In 1992-3, subjects from the original 12-year-old group (then aged 15 years), were reassessed. Ethical approval was obtained from the Medical Research Ethical Committee of The Queen's University of Belfast, and written consent was obtained from parents or guardians, and all participating subjects.

\section{Protocol}

As part of the test protocol, measurements of height, weight and skinfold thicknesses were made. Subjects, wearing light indoor clothing and no shoes, were weighed to the nearest $0 \cdot 1 \mathrm{~kg}$ using scales (Weylux balance model 424J London, UK; $160 \mathrm{~kg} \times 50 \mathrm{~g}$ ), and height was measured to the nearest $\mathrm{mm}$ using a free-standing, portable stadiometer (Holtain, Crymych, Wales, UK). Skinfold thicknesses were measured to the nearest $\mathrm{mm}$ using calipers at four sites (biceps, triceps, subscapular and suprailiac), and used to estimate body fatness (Durnin \& Rahaman, 1967). At follow-up, anthropometric data were obtained for 222 boys and 229 girls $(88 \%$ and $89 \%$ of original cohort respectively). Maturational stage at both time points was assessed using the Tanner index of pubertal development (Tanner, 1962). Nutritional data were collected from 225 boys and 230 girls (90\% and $89 \%$ of the original cohort respectively) by the diet history method with open-ended interview (van Staveren et al. 1985), and using food photographs to assist in the determination of portion sizes (Lee \& Cunningham, 1990). The diet history was used for two reasons. First, it has been shown, in this age group to provide more valid estimates of energy intake at the group level than weighed records (Livingstone et al. 1992). Second, given that a diet history can be obtained from a subject in approximately $1 \mathrm{~h}$, it was the most feasible and cost-effective method for obtaining dietary information from a school-based cohort of this size. Energy and nutrient intakes were calculated using a computerised database as previously described (Strain et al. 1994). The BMR of each subject was predicted using equations based on height and weight (Schofield et al. 1985). The same methods were used to assess diet and anthropometric status at both time points.

\section{Statistical analyses}

In an earlier paper (Strain et al. 1994), the advantages of using the median, rather than the arithmetic mean, to describe dietary intakes were outlined. Consequently, to maintain consistency in the present study, dietary data are summarised by medians, with the 25 th and 75 th percentiles included as a measure of variation. These percentiles are also used to describe body composition data. The Wilcoxon matched-pairs signed-ranks test was used to test for significant differences between median values at ages 12 and 15 years at a $5 \%$ significance level.

In the present study, daily energy or nutrient intake would be considered to track well over time if 12-year-old subjects with 'low', 'medium' or 'high' intakes maintained their ranking when 15-years-old. Basing the method on ranks was preferred to a method based on actual intakes because of its simplicity, its ability to show the rates of transitions between the three classes (low, medium or high) and also to deal with the problem of under- or overreporting of intakes. For example, in order to study the tracking of energy intakes (EI) of boys from aged 12 years to aged 15 years, the group of 225 boys aged 12 years was divided into three classes by EI: lowest $25 \%$ (L1); middle $50 \%$ (M1); highest $25 \%$ (H1). In effect, the classes were defined by the first and third empirical quartiles, and not by pre-determined fixed values. When aged 15 years, the group was divided into three similar classes: L2, M2 and $\mathrm{H} 2$. Using these two classifications, a $3 \times 3$ tracking matrix was constructed: the entry in a specific cell being the number of subjects belonging to the corresponding classes at age 12 and 15 years (examples are given in Fig. 1). Such a matrix provides a broad picture of the relative changes in intake of the group over the 3 year period. A matrix with relatively small off-diagonal elements provides evidence of 'good' tracking. The degree of tracking was summarised by a weighted kappa value (к) (Altman, 1991), calculated from the matrix. The interpretation of the $\kappa$ value obtained has been defined by Altman (1991) as follows: $\kappa<0 \cdot 20$, poor; $0 \cdot 21-0 \cdot 40$, fair; $0 \cdot 41-0 \cdot 60$, moderate; $0 \cdot 61-0 \cdot 8$, good; $0 \cdot 81-1.0$, very good. In addition, $95 \%$ confidence limits for $\kappa$ were calculated. This procedure was undertaken for intakes of energy, macronutrients and selected micronutrients.

\section{Results}

The physical characteristics and reported EI of the Young Hearts cohort at age 12 years and 15 years are presented in Table 1. The body weight and BMI of both boys and girls increased significantly $(P<0.001)$ with increasing age. At age 12 years and 15 years, the boys were heavier than the 50th centile value for weight for the equivalent UK population (2.7\% and $4.4 \%$ heavier respectively) (Department of Health, 1991). At both 12 years and 15 years, the girls were approximately $5.5 \%$ heavier than the population reference values (Department of Health, 1991). The median percentage body fat for boys decreased by $9 \%$ (in relative terms) from age 12 years to 15 years $(P<0.001)$, whereas that of the girls increased by a similar magnitude $(P<$ $0 \cdot 001$ ). In terms of maturational stage (not presented in 
(a)

\begin{tabular}{l|ccc} 
& $\mathrm{L} 2$ & $\mathrm{M} 2$ & $\mathrm{H} 2$ \\
\hline $\mathrm{L} 1$ & 21 & 26 & 9 \\
$\mathrm{M} 1$ & 27 & 61 & 25 \\
$\mathrm{H} 1$ & 8 & 26 & 22
\end{tabular}

к 0.18

\begin{tabular}{l|ccc} 
(c) & $\mathrm{L} 2$ & $\mathrm{M} 2$ & $\mathrm{H} 2$ \\
\hline $\mathrm{L} 1$ & 21 & 27 & 8 \\
$\mathrm{M} 1$ & 26 & 60 & 27 \\
$\mathrm{H} 1$ & 9 & 26 & 21
\end{tabular}

к 0.17 (b)

\begin{tabular}{l|ccc} 
& $\mathrm{L} 2$ & $\mathrm{M} 2$ & $\mathrm{H} 2$ \\
\hline L1 & 21 & 32 & 4 \\
M1 & 32 & 59 & 25 \\
H1 & 4 & 25 & 28 \\
& & & $\kappa 0.24$
\end{tabular}

in weight, BMI and \% body fat. However, when normalised for body weight $(\mathrm{kJ} / \mathrm{kg}$ per $\mathrm{d})$, EI reported by boys and girls was significantly lower at age 15 years than at age 12 years ( $P<0.001$, both sexes). The EI:predicted BMR ratio also decreased significantly over time in both groups, but the magnitude of change was greater for the girls than for the boys ( $P<0.001, P<0.05$ respectively). A fair degree of tracking was found over the study period for EI expressed per $\mathrm{kg}$ body weight ( $\kappa$ 0.37, 0.40 for boys and girls respectively), and for EI:BMR ( $\kappa 0.27,0.22$ for boys and girls respectively).

Over the 3 years, concomitant with the increase in reported $\mathrm{EI}$, median macronutrient intakes $(\mathrm{g} / \mathrm{d})$ of the boys increased significantly $(P<0.001$ for all macronutrients; Table 2). However, when expressed as a percentage contribution to EI, there were no significant changes in intakes of sugars or monounsaturated fatty acids reported at the group level. Small but statistically significant increases in intakes of \% energy derived from protein $(P<0.001)$ and polyunsaturated fatty acids $(P<0.05)$ were observed, whereas $\%$ energy contributed by total carbohydrate, total fat and saturated fatty acids in the boys' diets all decreased significantly over time $(P<0.001, P<0.05, P<0.001$ respectively). The reported polyunsaturated:saturated fatty acid ratio also increased $(P<0.001)$ from age 12 years (median 0.33) to age 15 years (median 0.39). Changes in the girls' intakes of macronutrients $(\mathrm{g} / \mathrm{d})$ were inconsistent. Despite the fact that median EI reported by the girls did not increase with age, intakes of protein $(\mathrm{g} / \mathrm{d})$ and carbohydrate $(\mathrm{g} / \mathrm{d})$ did increase significantly over time $(P<0 \cdot 05)$. Similarly to the boys, the median polyunsaturated:saturated fatty acid ratio and the median percentage contribution made by protein to EI also increased $(P<0.01, P<0.001$ respectively), whereas the percentage contributions made by carbohydrate and saturated fatty acids to EI were significantly lower $(P<0.001, P<0.01$ respectively) at age 15 years. Only ten boys and two girls reported drinking alcohol at age 12 years (data not shown). For consumers at age 15 years, the median (25th, 75 th percentile) intakes of

Table 1. Physical characteristics and energy intakes (EI) of the Young Hearts cohort of boys and girls at baseline (aged 12 years) and at followup (aged 15 years)

(Median values with 25th and 75th percentiles)

\begin{tabular}{|c|c|c|c|c|c|c|c|c|}
\hline & \multicolumn{4}{|c|}{ Boys } & \multicolumn{4}{|c|}{ Girls } \\
\hline & \multicolumn{2}{|c|}{ 12-years-old $(n$ 225) } & \multicolumn{2}{|c|}{ 15-years-old $(n$ 222)† } & \multicolumn{2}{|c|}{ 12-years-old $(n$ 230) } & \multicolumn{2}{|c|}{ 15-years-old (n 229)† } \\
\hline & Median & $\begin{array}{l}\text { 25th, } 75 \text { th } \\
\text { percentile }\end{array}$ & Median & $\begin{array}{l}\text { 25th, } 75 \text { th } \\
\text { percentile }\end{array}$ & Median & $\begin{array}{l}\text { 25th, 75th } \\
\text { percentile }\end{array}$ & Median & $\begin{array}{l}\text { 25th, } 75 \text { th } \\
\text { percentile }\end{array}$ \\
\hline Body weight $(\mathrm{kg})$ & $40 \cdot 9$ & $36 \cdot 1,48 \cdot 0$ & $59 \cdot 0^{\star \star \star}$ & $53 \cdot 2,67 \cdot 2$ & $43 \cdot 2$ & $37 \cdot 3,50 \cdot 0$ & $55 \cdot 4^{\star \star \star}$ & $50 \cdot 0,62 \cdot 1$ \\
\hline $\mathrm{BMI}\left(\mathrm{kg} / \mathrm{m}^{2}\right)$ & $18 \cdot 0$ & $17 \cdot 0,20 \cdot 4$ & $20 \cdot 3^{\star \star \star}$ & $18 \cdot 8,22 \cdot 3$ & $18 \cdot 8$ & $17 \cdot 1,21 \cdot 0$ & $21 \cdot 3^{\star \star \star}$ & $19 \cdot 4,23 \cdot 8$ \\
\hline Body fat (\%) & $18 \cdot 2$ & $15 \cdot 5,22 \cdot 4$ & $16 \cdot 6^{\star \star \star}$ & $14 \cdot 4,19 \cdot 7$ & $25 \cdot 2$ & $22 \cdot 6,29 \cdot 0$ & $27 \cdot 4^{\star \star \star}$ & $25 \cdot 0,29 \cdot 8$ \\
\hline El $(M J / d) \ddagger$ & $10 \cdot 9$ & $9 \cdot 1,13 \cdot 4$ & $13 \cdot 3^{\star \star *}$ & $11 \cdot 1,15 \cdot 1$ & $9 \cdot 0$ & $7 \cdot 7,10 \cdot 7$ & $9 \cdot 0$ & $7 \cdot 6,10 \cdot 6$ \\
\hline El $(\mathrm{kJ} / \mathrm{kg}$ per $\mathrm{d}) \ddagger$ & 262 & 215,333 & $217^{\star * \star}$ & 182,259 & 214 & 160,265 & $159^{\star \star \star}$ & 132,198 \\
\hline Energy requirements $(\mathrm{kJ} / \mathrm{kg}$ per $\mathrm{d}) \S$ & 237 & & 195 & & 196 & & 169 & \\
\hline El:BMR\| & 1.87 & $1 \cdot 55,2 \cdot 29$ & $1 \cdot 81^{*}$ & $1 \cdot 55,2 \cdot 10$ & 1.72 & $1 \cdot 40,2 \cdot 08$ & $1.51^{\star \star *}$ & $1 \cdot 28,1 \cdot 80$ \\
\hline
\end{tabular}

Group median values were significantly different from the group median values at 12 -years-old (Wilcoxon matched-pairs signed-ranks test): ${ }^{\star} P<0.05$, ${ }_{* \star *} P<0.001$.

† For energy intake, $n 225$ for boys and $n 230$ for girls.

$\ddagger$ Energy intakes were estimated by the diet history method with open-ended interview (van Staveren et al. 1985) using photographs to assist in the determination of portion sizes (Lee \& Cunningham, 1990) and a computerised database (Strain et al. 1994).

$\S$ Food and Agriculture Organization/World Health Organization/United Nations University (1985).

II BMR predicted from height and weight using equations of Schofield et al. (1985). 
Table 2. Intakes of macronutrients in the diets of the Young Hearts cohort of boys and girls at baseline (aged 12 years) and at follow-up (aged 15 years) $\dagger$

(Median values with 25th and 75th percentiles)

\begin{tabular}{|c|c|c|c|c|c|c|c|c|}
\hline & \multicolumn{4}{|c|}{ Boys ( $n$ 225) } & \multicolumn{4}{|c|}{ Girls ( $n$ 230) } \\
\hline & \multicolumn{2}{|c|}{ 12-years-old } & \multicolumn{2}{|c|}{ 15-years-old } & \multicolumn{2}{|c|}{ 12-years-old } & \multicolumn{2}{|c|}{ 15-years-old } \\
\hline & Median & $\begin{array}{l}\text { 25th, } 75 \text { th } \\
\text { percentile }\end{array}$ & Median & $\begin{array}{l}\text { 25th, 75th } \\
\text { percentile }\end{array}$ & Median & $\begin{array}{l}\text { 25th, } 75 \text { th } \\
\text { percentile }\end{array}$ & Median & $\begin{array}{l}\text { 25th, } 75 \text { th } \\
\text { percentile }\end{array}$ \\
\hline Protein (g/d) & $71 \cdot 2$ & $60 \cdot 2,85 \cdot 9$ & $92 \cdot 2^{\star \star \star}$ & $78 \cdot 8,107 \cdot 8$ & $59 \cdot 2$ & $49 \cdot 8,70 \cdot 8$ & $62 \cdot 6^{*}$ & $51 \cdot 7,74 \cdot 7$ \\
\hline$\%$ Energy from protein & $11 \cdot 0$ & $9 \cdot 9,12 \cdot 1$ & $11.9^{\star \star \star}$ & $10 \cdot 7,13 \cdot 1$ & $10 \cdot 8$ & $9 \cdot 7,12 \cdot 3$ & $11 \cdot 5^{\star \star \star}$ & $10 \cdot 3,12 \cdot 9$ \\
\hline Total $\mathrm{CHO}(\mathrm{g} / \mathrm{d})$ & $347 \cdot 6$ & $281 \cdot 3,429 \cdot 4$ & $404 \cdot 1^{\star \star \star}$ & $337 \cdot 0,466 \cdot 4$ & 281.4 & $239 \cdot 6,337 \cdot 0$ & $277 \cdot 2^{\star}$ & $228 \cdot 4,319 \cdot 6$ \\
\hline$\%$ Energy from $\mathrm{CHO}$ & 49.4 & $46 \cdot 7,52 \cdot 1$ & $47 \cdot 7^{\star \star *}$ & $44 \cdot 6,50 \cdot 0$ & $49 \cdot 3$ & $46 \cdot 4,52 \cdot 6$ & $47 \cdot 9^{\star \star \star}$ & $45 \cdot 0,50 \cdot 6$ \\
\hline Sugars $(g / d)$ & $137 \cdot 4$ & $110 \cdot 9,182 \cdot 6$ & $158 \cdot 8^{\star \star \star}$ & $123 \cdot 7,200 \cdot 1$ & $118 \cdot 8$ & $94 \cdot 6,149 \cdot 4$ & $115 \cdot 0$ & $90 \cdot 0,150 \cdot 0$ \\
\hline$\%$ Energy from sugars & $20 \cdot 3$ & $16 \cdot 7,23 \cdot 3$ & $19 \cdot 4$ & $16 \cdot 3,22 \cdot 6$ & $20 \cdot 6$ & $17 \cdot 7,23 \cdot 7$ & $20 \cdot 3$ & $17 \cdot 1,23 \cdot 8$ \\
\hline Total fat $(\mathrm{g} / \mathrm{d})$ & $112 \cdot 0$ & $89 \cdot 3,140 \cdot 1$ & $130 \cdot 1^{\star \star \star}$ & $110.5,155.3$ & $93 \cdot 0$ & $75 \cdot 8,114 \cdot 2$ & $92 \cdot 9$ & $76.5,111.4$ \\
\hline$\%$ Energy from fat & $39 \cdot 0$ & $36 \cdot 0,41 \cdot 6$ & $38 \cdot 2^{*}$ & $35 \cdot 2,40 \cdot 8$ & 38.4 & $35 \cdot 7,41 \cdot 8$ & $39 \cdot 1$ & $36 \cdot 3,41 \cdot 6$ \\
\hline SFA $(g / d)$ & $45 \cdot 1$ & $35 \cdot 3,58 \cdot 3$ & $51 \cdot 8^{\star \star \star}$ & $41 \cdot 0,61 \cdot 7$ & $35 \cdot 7$ & $28 \cdot 4,46 \cdot 2$ & 34.8 & $28 \cdot 4,42 \cdot 6$ \\
\hline$\%$ Energy from SFA & $15 \cdot 9$ & $13 \cdot 8,17 \cdot 8$ & $14 \cdot 4^{\star \star *}$ & $12 \cdot 9,16 \cdot 6$ & $15 \cdot 1$ & $13 \cdot 3,17 \cdot 1$ & $14 \cdot 3^{\star \star}$ & $12 \cdot 8,16 \cdot 6$ \\
\hline MUFA $(g / d)$ & $36 \cdot 3$ & $28 \cdot 1,46 \cdot 3$ & $43 \cdot 5^{\star \star \star \star}$ & $35.4,53 \cdot 2$ & 29.4 & $24 \cdot 1,36 \cdot 5$ & 29.9 & $24 \cdot 0,35 \cdot 6$ \\
\hline \% Energy from MUFA & $12 \cdot 7$ & $11.5,13.7$ & $12 \cdot 5$ & $11 \cdot 2,13 \cdot 9$ & $12 \cdot 3$ & $11 \cdot 1,13 \cdot 4$ & $12 \cdot 3$ & $11 \cdot 0,13 \cdot 7$ \\
\hline PUFA (g/d) & $15 \cdot 4$ & $11 \cdot 5,20 \cdot 2$ & $19 \cdot 6^{\star \star *}$ & $14 \cdot 6,25 \cdot 8$ & 14.4 & $10 \cdot 6,18 \cdot 9$ & 14.9 & $10 \cdot 8,18 \cdot 9$ \\
\hline$\%$ Energy from PUFA & $5 \cdot 1$ & $4 \cdot 2,6 \cdot 4$ & $5 \cdot 7^{*}$ & $4 \cdot 4,7 \cdot 1$ & 5.9 & $4 \cdot 7,7 \cdot 3$ & $6 \cdot 0$ & $4 \cdot 8,7 \cdot 8$ \\
\hline $\mathrm{P}: \mathrm{S}$ ratio & 0.33 & $0.24,0.47$ & $0.39^{* * *}$ & $0.29,0.53$ & 0.38 & $0.29,0.53$ & $0.43^{\star *}$ & $0.31,0.57$ \\
\hline
\end{tabular}

$\mathrm{CHO}$, carbohydrate; SFA, saturated fat; MUFA, monounsaturated fat; PUFA, polyunsaturated fat; P:S ratio, polyunsaturated:saturated fatty acid ratio.

Group median values were significantly different from the group median values at 12 -years-old (Wilcoxon matched-pairs signed-ranks test): ${ }^{\star} P<0.05$, ${ }^{\star} P<0.01$, ${ }^{\star * \star} P<0.001$

† Macronutrient intakes were estimated by the diet history method with open-ended interview (van Staveren et al. 1985) using photographs to assist in the determination of portion sizes (Lee \& Cunningham, 1990) and a computerised database (Strain et al. 1994).

ethanol for boys $(n 84)$ and girls $(n 68)$ were $8.8(4.5,14.8)$ $\mathrm{g}$ and $4.3(2 \cdot 6,9.9) \mathrm{g}$ respectively.

While at the group level, some significant changes in energy and macronutrient intakes were observed, it is notable that the tracking of energy and macronutrient intakes at the individual level was only poor to fair. Fig. 1 shows the $3 \times 3$ matrices and $\kappa$ obtained for tracking of reported EI and \% energy derived from fat. The tracking of EI (MJ/d) was poor for boys, and fair for girls. The matrices demonstrate substantial drift between the classes of intake. For example, $46 \%$ of boys who reported EI in the lowest intake category (L1) at age 12 years, moved into the medium intake category (M2) when assessed at age 15 years, and $16 \%$ of the cohort moved from the L1 category

Table 3. Tracking of energy and macronutrient intakes, as estimated by weighted kappa values ( $\mathrm{\kappa})$, in the diets of boys and girls in the Young Hearts cohort from baseline (aged 12 years) to follow-up (aged 15 years)

\begin{tabular}{lcc}
\hline & \multicolumn{2}{c}{$\kappa^{*}$} \\
\cline { 2 - 3 } & Boys $(n$ 225) & Girls $(n$ 230) \\
\hline El $(\mathrm{MJ})$ & 0.18 & 0.24 \\
El:BMR & 0.27 & 0.22 \\
El/kg body weight & 0.37 & 0.40 \\
Protein (g) & 0.16 & 0.25 \\
\% Energy from protein & 0.25 & 0.28 \\
Carbohydrate $(\mathrm{g})$ & 0.17 & 0.17 \\
\% Energy from carbohydrate & 0.16 & 0.17 \\
Sugars (g) & 0.19 & 0.14 \\
\% Energy from sugars & 0.18 & 0.09 \\
Fat (g) & 0.18 & 0.20 \\
\% Energy from fat & 0.17 & 0.09 \\
\hline
\end{tabular}

El, energy intake.

* Weighted kappa values ( $\kappa$ ) were calculated as described on p. $542.95 \% \mathrm{Cl}$ were $\pm 0 \cdot 1$ throughout. into the highest intake category (H2). Substantial migration between categories was also apparent for boys' and girls' reported intakes of $\%$ energy derived from fat. The $\kappa$ values for energy and macronutrients are given in Table 3. With the exception of percentage energy derived from protein $(\kappa$ $0 \cdot 25$, fair tracking), the tracking of EI and macronutrients in boys from age 12 to age 15 years was poor $(\kappa<0 \cdot 20)$. For girls, EI $(\mathrm{MJ} / \mathrm{d})$ and protein intake (g/d and \% energy) exhibited a fair degree of tracking, but this was not evident for other macronutrients. When expressed as \% EI, particularly poor tracking was evident for the girls'

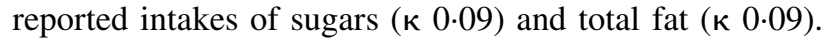

Table 4 presents reported intakes of selected micronutrients by the Young Hearts cohort at age 12 years and at follow-up (aged 15 years). The median micronutrient densities (mg/MJ per d or $\mu \mathrm{g} / \mathrm{MJ}$ per d) of the boys' diets did not remain consistent with increasing age. Over time, the boys' diets became slightly, but significantly, richer in $\mathrm{Fe}(P<0.05)$, vitamin $\mathrm{B}_{6}(P<0.001)$ and total folate $(P<0.001)$. In contrast, $\mathrm{Ca}(P<0.01)$ and riboflavin $(P<0.001)$ densities fell with increasing age. No significant changes in intakes per MJ of the other micronutrients were observed in the boys' diets. The boys' median intakes of total folate $(\mu \mathrm{g} / \mathrm{MJ})$ were lower than estimated average requirements (EAR, expressed as $\mu \mathrm{g} / \mathrm{MJ}$ (Department of Health, 1991)), both at age 12 years $(-28.4 \%)$ and at age 15 years $(-1.5 \%)$. Median intakes of all other micronutrients exceeded the EAR, when expressed in terms of nutrient density. Similarly to the boys, the girls' diets became richer in folate $(P<0.01)$ over time, and poorer in $\mathrm{Ca}$ and riboflavin (both $P<$ 0.001). The girls' intakes of thiamin (mg/MJ per d) also decreased with increasing age. No changes were observed in median daily intakes of Fe, vitamins $\mathrm{A}, \mathrm{C}$ and $\mathrm{D}$ per MJ 
Table 4. Intakes of selected micronutrients in the diets of the Young Hearts cohort of boys and girls at baseline (aged 12 years) and at follow-up (aged 15 years) $\dagger$

(Median values with 25th and 75th percentiles)

\begin{tabular}{|c|c|c|c|c|c|c|c|c|c|}
\hline \multirow[b]{3}{*}{ Micronutrient } & \multirow[b]{3}{*}{ Unit } & \multicolumn{4}{|c|}{ Boys ( $n$ 225) } & \multicolumn{4}{|c|}{ Girls (n 230) } \\
\hline & & \multicolumn{2}{|c|}{ 12-years-old } & \multicolumn{2}{|c|}{ 15-years-old } & \multicolumn{2}{|c|}{ 12-years-old } & \multicolumn{2}{|c|}{ 15-years-old } \\
\hline & & Median & $\begin{array}{l}\text { 25th, } 75 \text { th } \\
\text { percentile }\end{array}$ & Median & $\begin{array}{l}\text { 25th, } 75 \text { th } \\
\text { percentile }\end{array}$ & Median & $\begin{array}{l}\text { 25th, } 75 \text { th } \\
\text { percentile }\end{array}$ & Median & $\begin{array}{l}\text { 25th, 75th } \\
\text { percentile }\end{array}$ \\
\hline \multirow[t]{2}{*}{ Iron } & $\mathrm{mg} / \mathrm{d}$ & $11 \cdot 0$ & $9 \cdot 3,13 \cdot 8$ & $14 \cdot 2^{* \star \star}$ & $11 \cdot 5,16 \cdot 4$ & 9.73 & $8.0,11.9$ & 9.9 & $8 \cdot 4,11 \cdot 8$ \\
\hline & mg/MJ per d & 1.00 & $0.92,1.11$ & $1.05^{\star}$ & 0.93 & 1.06 & $0.94,1.19$ & 1.08 & 1.00 \\
\hline \multirow{2}{*}{ Calcium } & $\mathrm{mg} / \mathrm{d}$ & 1013 & 782,1274 & $1083^{\star \star \star}$ & 849,1384 & 819 & 654,1036 & $757^{\star \star}$ & 575,963 \\
\hline & $\mathrm{mg} / \mathrm{MJ}$ per $\mathrm{d}$ & $88 \cdot 6$ & $73 \cdot 6,106 \cdot 8$ & $84 \cdot 1^{\star *}$ & $69 \cdot 5,101 \cdot 7$ & $89 \cdot 0$ & $70 \cdot 6,112 \cdot 1$ & $82 \cdot 4^{\star \star \star}$ & $65 \cdot 8,102 \cdot 1$ \\
\hline \multirow[t]{2}{*}{ Thiamin } & $\mathrm{mg} / \mathrm{d}$ & 1.54 & $1 \cdot 17,1 \cdot 83$ & $1 \cdot 75^{\star \star *}$ & $1 \cdot 42,2 \cdot 14$ & $1 \cdot 22$ & $0.97,1.54$ & $1.06^{\star * *}$ & $0.86,1.40$ \\
\hline & $\mathrm{mg} / \mathrm{MJ}$ per $\mathrm{d}$ & 0.13 & $0.11,0.16$ & 0.14 & $0.11,0.17$ & 0.13 & $0.11,0.16$ & $0 \cdot 12^{\star \star *}$ & $0 \cdot 10,0 \cdot 15$ \\
\hline \multirow[t]{2}{*}{ Riboflavin } & $\mathrm{mg} / \mathrm{d}$ & 1.98 & $1.47,2.56$ & $2 \cdot 15^{\star \star}$ & $1 \cdot 58,2 \cdot 70$ & 1.56 & $1.20,1.92$ & $1 \cdot 21^{\star \star \star}$ & $0 \cdot 86,1 \cdot 60$ \\
\hline & $\mathrm{mg} / \mathrm{MJ}$ per $\mathrm{d}$ & 0.18 & $0.14,0.22$ & $0 \cdot 16^{\star \star \star}$ & $0.12,0.21$ & 0.17 & $0.14,0.21$ & $0.13^{\star \star *}$ & $0 \cdot 10,0 \cdot 17$ \\
\hline \multirow{2}{*}{ Vitamin $B_{6}$} & $\mathrm{mg} / \mathrm{d}$ & 1.45 & $1 \cdot 19,1 \cdot 75$ & $1 \cdot 86^{\star \star \star}$ & $1 \cdot 58,2 \cdot 22$ & $1 \cdot 28$ & $1.05,1.55$ & 1.29 & $1.07,1.53$ \\
\hline & $\mathrm{mg} / \mathrm{MJ}$ per d & 0.13 & $0 \cdot 12,0.15$ & $0 \cdot 15^{\star \star \star}$ & $0.13,0.17$ & 0.14 & $0.12,0.16$ & 0.14 & $0 \cdot 13,0 \cdot 16$ \\
\hline \multirow[t]{2}{*}{ Total folate } & $\mu \mathrm{g} / \mathrm{d}$ & 133 & 106,160 & $169^{\star \star \star}$ & 138,215 & 116 & 97,145 & $128^{\star \star}$ & 101,158 \\
\hline & $\mu \mathrm{g} / \mathrm{MJ}$ per $\mathrm{d}$ & $11 \cdot 6$ & $9 \cdot 9,14.5$ & $12 \cdot 8^{\star \star \star}$ & $10 \cdot 7,15 \cdot 5$ & $12 \cdot 8$ & $10 \cdot 4,15 \cdot 6$ & $13 \cdot 9^{\star \star}$ & $10 \cdot 8,17 \cdot 5$ \\
\hline \multirow[t]{2}{*}{ Vitamin A } & $\mu \mathrm{g} R E / \mathrm{d}$ & 638 & 483,924 & $790^{\star \star \star}$ & 580,1062 & 567 & 413,816 & 602 & 448,810 \\
\hline & $\mu \mathrm{g}$ RE/MJ per d & $60 \cdot 4$ & $45 \cdot 3,80 \cdot 9$ & $61 \cdot 2$ & $48 \cdot 1,79 \cdot 1$ & $62 \cdot 3$ & $46 \cdot 5,81 \cdot 5$ & $67 \cdot 7$ & $50 \cdot 3,88 \cdot 0$ \\
\hline \multirow[t]{2}{*}{ Vitamin C } & $\mathrm{mg} / \mathrm{d}$ & $61 \cdot 7$ & $44 \cdot 0,89.5$ & $74 \cdot 8^{\star \star \star}$ & $53 \cdot 3,104 \cdot 8$ & $68 \cdot 2$ & $44 \cdot 0,96 \cdot 2$ & $64 \cdot 6$ & $40 \cdot 9,99 \cdot 8$ \\
\hline & $\mathrm{mg} / \mathrm{MJ}$ per d & 5.50 & $4.23,7.59$ & $5 \cdot 69$ & $3 \cdot 92,8 \cdot 12$ & $7 \cdot 2$ & $4.89,10 \cdot 19$ & $6 \cdot 81$ & $4.30,10.99$ \\
\hline \multirow[t]{2}{*}{ Vitamin D } & $\mu \mathrm{g} / \mathrm{d}$ & 1.13 & $0.61,2.09$ & 1.22 & $0.49,2.29$ & 1.13 & $0.57,1.97$ & 1.25 & $0 \cdot 50,2 \cdot 24$ \\
\hline & $\mu \mathrm{g} / \mathrm{MJ}$ per $\mathrm{d}$ & 0.11 & $0.06,0.19$ & 0.08 & $0.04,0.19$ & 0.13 & $0.07,0.21$ & 0.14 & $0.06,0.24$ \\
\hline
\end{tabular}

RE, retinol equivalents.

Group Median values were significantly different from the group median values at 12 -years-old (Wilcoxon matched-pairs signed-ranks test): $P<0.05,{ }^{\star \star} P<0.01$, ${ }^{\star * *} P<0.001$.

$\uparrow$ Micronutrient intakes were estimated by the diet history method with open-ended interview (van Staveren et al. 1985) using photographs to assist in the determination of portion sizes (Lee \& Cunningham, 1990) and a computerised database (Strain et al. 1994).

in the girls' diets. The shortfall in total folate intakes of the girls, relative to EAR $(\mu \mathrm{g} / \mathrm{MJ})$, was substantial at both time points $(-34.0 \%,-18.2 \%$ at 12 years and 15 years respectively). Moreover, median $\mathrm{Fe}$ intakes (mg/MJ) reported by the girls were lower than the EAR (mg/MJ) at age 12 years $(-28.4 \%)$ and at age 15 years $(-16 \cdot 3 \%)$.

Table 5. Tracking of micronutrient intakes as estimated by weighted kappa values ( $\kappa)$, in the diets of boys and girls of the Young Hearts cohort from baseline (aged 12 years) to follow-up (aged 15 years)

\begin{tabular}{lccc}
\hline & & \multicolumn{2}{c}{$\kappa^{*}$} \\
\cline { 3 - 4 } Micronutrient & Unit & Boys $(n 225)$ & Girls $(n 230)$ \\
\hline Iron & $\mathrm{mg}$ & 0.17 & 0.21 \\
& $\mathrm{mg} / \mathrm{MJ}$ & 0.21 & 0.22 \\
Calcium & $\mathrm{mg}$ & 0.25 & 0.22 \\
& $\mathrm{mg} / \mathrm{MJ}$ & 0.31 & 0.29 \\
Thiamin & $\mathrm{mg}$ & 0.24 & 0.18 \\
& $\mathrm{mg} / \mathrm{MJ}$ & 0.30 & 0.25 \\
Riboflavin & $\mathrm{mg}$ & 0.29 & 0.23 \\
& $\mathrm{mg} / \mathrm{MJ}$ & 0.29 & 0.19 \\
Vitamin $\mathrm{B}_{6}$ & $\mathrm{mg}$ & 0.17 & 0.16 \\
& $\mathrm{mg} / \mathrm{MJ}$ & 0.14 & 0.21 \\
Total folate & $\mu \mathrm{g}$ & 0.24 & 0.17 \\
Vitamin $\mathrm{A}$ & $\mu \mathrm{g} / \mathrm{MJ}$ & 0.31 & 0.26 \\
& $\mu \mathrm{RE}$ & 0.23 & 0.17 \\
Vitamin C & $\mu \mathrm{RE} / \mathrm{MJ}$ & 0.20 & 0.21 \\
Vitamin $\mathrm{D}$ & $\mathrm{mg}$ & 0.19 & 0.18 \\
& $\mathrm{mg} / \mathrm{MJ}$ & 0.24 & 0.20 \\
& $\mu \mathrm{g}$ & 0.19 & 0.23 \\
& $\mu \mathrm{g} / \mathrm{MJ}$ & 0.13 & 0.19 \\
\hline
\end{tabular}

$\mathrm{RE}$, retinol equivalent.

* Weighted kappa values ( $\kappa$ ) were calculated as described on p. $542.95 \% \mathrm{Cl}$ were \pm 0.1 throughout.
Median intakes of the other micronutrients reported in this study exceeded EAR. The weighted $\kappa$ values obtained for the micronutrient intakes are summarised in Table 5. Again, while there were some significant changes at the group level over time in both sexes, micronutrient intakes exhibited only poor to fair tracking. For intakes of $\mathrm{Fe}(\mathrm{mg} /$ $\mathrm{MJ}), \mathrm{Ca}(\mathrm{mg} / \mathrm{MJ})$, thiamin $(\mathrm{mg} / \mathrm{MJ})$, and total folate $(\mu \mathrm{g} /$ MJ), a fair degree of tracking (к $0 \cdot 21-0 \cdot 31)$ was observed for boys and girls. Intakes of riboflavin and vitamin $\mathrm{C}$, adjusted for EI, exhibited a fair degree of tracking for boys,

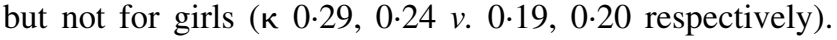
Poor tracking was observed for boys' intakes of vitamin $B_{6}$ $(\mathrm{mg} / \mathrm{MJ})$ and vitamin $\mathrm{A}(\mu \mathrm{g}$ retinol equivalents/MJ) (к $0 \cdot 14$ and 0.20 respectively), whereas for girls this was marginally stronger (both к $0 \cdot 21$ ). Vitamin D intakes, adjusted for EI, exhibited only poor tracking in both sexes.

\section{Discussion}

While at the group level there were some significant changes in nutrient intakes in the Young Hearts cohort between the ages of 12 years and 15 years, it is notable that tracking for most nutrients was only poor to fair. Intuitively, this is not an unexpected finding, as adolescence is a time characterised by considerable physical, cognitive and psychosocial change. However, to date, the evidence for tracking, or otherwise, of nutrient intakes in this age group has been somewhat scarce and inconsistent. In contrast, several studies of younger children have reported fair to moderate tracking of certain nutrients. One study, which examined the extent of tracking of 
nutrients in the diets of 181 children initially aged 45-60 months, reported tracking coefficients ranging from $0 \cdot 19$ for polyunsaturated fatty acids to 0.71 for $\mathrm{Ca}$ (Stein et al. 1991). Despite substantial day-to-day variation in intake, the authors concluded that the underlying diets, assessed using repeated $24 \mathrm{~h}$ recalls, of the pre-school children did track well over time. A later study, which used a series of $3 \mathrm{~d}$ estimated food diaries to assess the diets of seventythree children from 3-4 years to 7-8 years of age, also found evidence of nutrient tracking in the pre-school years (Singer et al. 1995). However, as the subjects in these studies were surveyed at young ages when diet is highly likely to be controlled by parents or guardians, this is probably to be expected.

Existing evidence for the phenomenon of nutrient tracking in older children and adolescents is less consistent. For example, Boulton et al. (1995) reported tracking of dietary energy, fat and $\mathrm{Ca}$ in an Australian cohort from the age of 1 to 15 years. Diets in that cohort were assessed using $4 \mathrm{~d}$ weighed records. Tracking was relatively consistent for boys' $\mathrm{Ca}$ intakes from early and midchildhood, through adolescence. It was also apparent that children who were 'big eaters' at a young age remained so, but that those who had lower energy intakes when younger became more evenly spread across the distribution of intakes over time. Another study, the Amsterdam Growth and Health Study, for which nutrient intake was assessed using the diet history method, also described fair to moderate tracking of $\mathrm{Ca}$ intakes in males and females over a 12-year period (Welten et al. 1997). More data from the same cohort described 'tracking coefficients' for \% energy derived from fat (males 0.33 , females 0.04 ), protein (males 0.32, females 0.04), and carbohydrate (males 0.19, females 0.06) (Twisk et al. 1997b). These coefficients, computed from data obtained in the first dietary assessment undertaken at age 13 years and the mean of the last two assessments made at age 27-29 years, suggest poor to fair tracking of macronutrient intakes in males, but poor tracking in females.

It is difficult to draw direct comparisons between these studies and the present study, owing to substantial differences in dietary intake methodology, methods of calculating tracking coefficients, ages of the populations studied, and time lapses between periods of assessment. In the present study, tracking was only poor to fair between the ages of 12 and 15 years, demonstrating a marked drift of individuals between classes of intake for most nutrients. In other words, although there were statistically significant changes in intakes of many nutrients at the group level over time, this was not because all subjects reported intakes which increased or decreased proportionately with increasing age.

The lowest $\kappa$ values in the present cohort were found in the girls for $\%$ energy derived from fat (к 0.09) and \% energy derived from sugars ( $\kappa$ 0.09). These values indicate $3 \times 3$ matrices with relatively large off-diagonal elements (poor tracking), and are somewhat lower than those reported by the boys ( $\kappa 0.17$ and $\kappa 0.18$ respectively). There are two possible explanations for the particularly poor tracking of these specific nutrients in girls: either the values are an artifact related to 'mis-reporting' of intake, or they reflect real changes in nutrient consumption patterns with increasing age. As previously described (Strain et al. 1994), a subject's intake was considered to be implausible (or 'mis-reported') if EI:BMR was less than 1.14 ('underreporting') or greater than 2.5 ('over-reporting') (cut-offs based on equations derived by Goldberg et al. 1991). In the present study, less than $6 \%$ of boys were considered to be 'under-reporters' at both time points. However, $8.3 \%$ of the girls reported implausibly low EI at age 12 years, and this increased to $13.5 \%$ at age 15 years. Possible 'overreporting' was more common in boys with $19.6 \%$ and $7.2 \%$ of boys, at age 12 and at age 15 years respectively, reporting implausibly high EI. In the girls, $8.3 \%$ and $3.9 \%$ of the cohort (at age 12 years and 15 years respectively) were considered to be 'over-reporters'.

The higher proportion of girls 'under-reporting' at age 15 years could partially explain the low tracking coefficients obtained for \% energy from fat and sugars. It has been suggested that adult 'low-energy-reporters' may selectively 'mis-report' specific nutrients or foods. For example, several studies have shown that subjects who were considered to be low-energy-reporters appeared to obtain less energy from fat (Bingham et al. 1995; Crawley \& Shergill-Bonner, 1995) and sugars (Pryer et al. 1995), than those who were not low-energy-reporters. It is possible that older girls, who are more likely to be preoccupied with body image (Crawley \& Summerbell, 1997), may be less inclined to accurately report foods higher in fat and sugar, which they perceive to be 'unhealthy'. This would also help to explain the fact that, at the group level, the reported EI in girls did not increase over time, despite increases in height, weight and \% body fat. The highest $\kappa$ values in the present cohort were obtained for EI when expressed relative to body weight (boys $\kappa ~ 0.37$, girls $\kappa ~ 0.40$ ). These values, which are approaching moderate tracking, suggest that to some degree, subjects were more inclined to maintain their rank over time for EI per kg body weight than they were for other nutrients. Work by Price et al. (1997) has demonstrated that adults who reported a lower EI on one occasion were likely to do so on another occasion. As yet however, there is no substantial evidence for this phenomenon in adolescents and further work is required in this area.

There are several possible explanations for the relatively low $\kappa$ values obtained for the majority of the nutrients in the present study. First, the data may simply indicate that adolescence is, indeed, associated with rapidly changing, and erratic, patterns of nutrient intake. Teenagers take increasing control of what, when and where they eat and typically consume a greater proportion of their total food outside the home. Furthermore, intense awareness and concern about body shape and appearance often prompt attempts to alter body size via limiting food intake and other techniques. Second, it is possible that the diet history method is not a suitably robust tool for assessing tracking of nutrient intakes in a cohort of this nature. Järvinen et al. (1993) have suggested that the reproducibility of the diet history method is acceptable for epidemiological studies in adults, where eating patterns remain relatively stable. However, there are currently few data concerning the reproducibility of any of the routinely used dietary assessment methods in the age group involved in this study. Although the diet history has been shown to provide 
good validity at the group level in older children and adolescents, it is prone to significant problems of precision at the individual level (Livingstone et al. 1992). Moreover, since it measures memory and perception of usual diet and is vulnerable to socially desirable responding (Livingstone \& Robson, 2000), it is entirely possible that the degree of memory and motivation required to complete a diet history may change substantially over time, thereby contributing to the apparently poor tracking observed in this study. However, it seems unlikely that any other method would have provided evidence of stronger tracking in this cohort. One alternative, the weighed record, is particularly prone to increasing negative bias with increasing age in adolescents (Livingstone et al. 1992). Furthermore, $24 \mathrm{~h}$ recalls and food frequency questionnaires have not been subjected to rigorous validity checks in younger age groups. Thus it remains unclear whether they would be any more suitable than the diet history for assessing tracking of nutrient intakes in adolescence.

In general, adolescent diets are perceived to be relatively high in fat and/or sugars and low in vitamins and minerals. At both 12 and 15 years of age, the diets reported by boys and girls were higher in total fat and saturated fat than currently recommended (Department of Health, 1991). However, it is important to note that the median intakes of these nutrients were of a similar magnitude to the mean intakes observed in British adults (Gregory et al. 1990), suggesting that diets with apparently 'unfavourable' lipid profiles are not a phenomenon exclusive to adolescents. Moreover, when the vitamin and mineral intakes of the cohort were expressed as nutrient densities and compared with EAR (Department of Health, 1991), also expressed in terms of nutrient densities, there was apparently less cause for concern than is often thought. For most vitamins and minerals, median intakes were in excess of average requirements. However the data do indicate that, although diets became more rich in total folate over time, the median intakes of boys and girls remained lower than the current EAR (Department of Health, 1991). The girls' Fe intakes (mg/MJ) were also lower than the current EAR. Low $\mathrm{Fe}$ and folate intakes have previously been reported in Irish girls aged 12 years and 15 years (Hurson \& Corish, 1997). However, as these data were expressed in absolute values, direct comparisons with the present study are difficult. It is evident from the analysis of the current data, that provision of absolute intakes of energy and nutrients does not give an informed base with which to judge properly the nutritional quality of adolescent diets. Therefore, it is recommended that future studies in this area should give serious consideration to describing nutrient intake in terms of nutrient density.

Overall, the data presented in the current study suggest that individual dietary patterns reported at 12 years of age are unlikely to be predictive of energy and nutrient intakes reported at age 15 years. However, these observations are based on the assumption that the reported intakes are representative at both time points. Given the apparent lack of tracking of nutrient intakes in this age group, it is clear that individual subjects cannot, and should not, be targeted for dietary intervention, based solely on data obtained at 12 years of age.

\section{Acknowledgements}

The first two phases of the Young Hearts Project were supported by the Northern Ireland Chest, Heart and Stroke Association. The Project Team would also like to thank the British Heart Foundation and the Wellcome Trust for their support of Young Hearts III. The authors would like to thank the subjects who participated in the project, and all fieldworkers who collected the dietary data (Hilary Morrison, Elizabeth Archer, Siobhan Higgins and Dara Morgan).

\section{References}

Adamson A, Rugg-Gunn AJ, Butler TJ, Appleton DR \& Hackett AF (1992) Nutritional intake, height and weight of 11-12 year old Northumbrian children in 1990 compared with information obtained in 1980. British Journal of Nutrition 68, 543-563.

Altman DG (1991) In Practical Studies for Medical Research London: Chapman \& Hall, pp. 404-407.

Anderson AS, Macintyre S \& West P (1994) Dietary patterns among adolescents in the west of Scotland. British Journal of Nutrition 71, 111-122.

Bingham SA, Cassidy A, Cole TJ, Welch A, Runswick SA, Black AE, Thurnham D, Bates C, Khaw KT, Key TJA \& Day NE (1995) Validation of weighed records and other methods of dietary assessment using the $24 \mathrm{~h}$ urine nitrogen technique and other biological markers. British Journal of Nutrition 73, 531550.

Boreham C, Savage JM, Primrose D, Cran G \& Strain J (1993) Coronary risk factors in schoolchildren. Archives of Disease in Childhood 68, 182-186.

Boulton TJC, Margarey AM \& Cockington RA (1995) Tracking of serum lipids and dietary energy, fat and calcium intake from 1 to 15 years. Acta Paediatrica 84, 1050-1055.

Crawley HF (1993) The energy, nutrient and food intakes of teenagers aged 16-17 years in Britain 1. Energy, macronutrients and non-starch polysaccharides. British Journal of Nutrition 70, 15-26.

Crawley H \& Shergill-Bonner R (1995) The nutrient and food intakes of 16-17 year old female dieters. Journal of Human Nutrition and Dietetics 8, 25-34.

Crawley H \& Summerbell C (1997) Feeding frequency and BMI among teenagers aged 16-17 years. International Journal of Obesity and Related Metabolic Disorders 21, 159-161.

Department of Health (1991) In Dietary Reference Values for Food Energy and Nutrients for the United Kingdom London: HM Stationery Office.

Durnin JVG \& Rahaman MM (1967) The assessment of the amount of fat in the human body by measurements of skinfold thickness. British Journal of Nutrition 21, 681-689.

Food and Agriculture Organization/World Health Organization/ United Nations University (1985) In Energy and Protein Requirements. WHO Technical Report Series no. 724. Geneva: WHO.

Goldberg GR, Black AE, Jebb SA, Cole TJ, Murgatroyd PR, Coward WA \& Prentice AM (1991) Critical evaluation of energy intake data using fundamental principles of energy physiology: 1. Derivation of cut-off limits to identify underrecording. European Journal of Clinical Nutrition 45, 569-581.

Gregory J, Foster K, Tyler H \& Wiseman M (1990) In The Dietary and Nutritional Survey of British Adults. London: HM Stationery Office.

Hurson M \& Corish C (1997) Evaluation of lifestyle, food consumption and nutrient intake patterns among Irish teenagers. Irish Journal of Medical Science 166, 225-230. 
Järvinen R, Seppänen R \& Knekt P (1993) Short-term and longterm reproducibility of dietary history interview data. International Journal of Epidemiology 22, 520-527.

Kelder SH, Perry CL, Klepp K-I \& Lytle LL (1994) Longitudinal tracking of adolescent smoking, physical activity and food choice behaviors. American Journal of Public Health 84, 11211126.

Lauer RM, Lee J \& Clarke WR (1988) Factors affecting the relationship between childhood and adult cholesterol levels: the Muscatine Study. Pediatrics 82, 309-318.

Lee P \& Cunningham K (1990) In Irish National Nutrition Survey. Dublin: The Irish Nutrition and Dietetic Institute.

Livingstone MBE, Prentice AM, Coward WA, Strain JJ, Black AE, Davies PSW, Stewart CM, McKenna PG \& Whitehead RG (1992) Validation of estimates of energy intake by weighed dietary record and diet history in children and adolescents. American Journal of Clinical Nutrition 56, 29-35.

Livingstone MBE \& Robson PJ, (2000) Measurement of dietary intake in children. Proceedings of the Nutrition Society (In the Press).

Porkka KVK, Viikari JSA, Taimela S, Dahl M \& Akerblom HK (1994) Tracking and predictiveness of serum lipid and lipoprotein measurements in childhood: a 12-year follow-up. The Cardiovascular Risk in Young Finns Study. American Journal of Epidemiology 140, 1096-1110.

Price GM, Paul AA, Cole TJ \& Wadsworth EJ (1997) Characteristics of the low-energy reporters in a longitudinal national dietary survey. British Journal of Nutrition 77, 833851.

Pryer J, Brunner E, Elliott P, Nichols R, Dimond H \& Marmot M (1995) Who complied with COMA dietary fat recommendations among a nationally representative sample of British adults in 1986-7 and what did they eat? European Journal of Clinical Nutrition 49, 718-728.

Raitakari OT, Porkka KVK, Räsänen L, Rönnemaa T \& Viikari JS (1994) Clustering and 6-year cluster-tracking of serum total cholesterol, HDL-cholesterol and diastolic blood pressure in children and young adults. The Cardiovascular Risk in Young Finns Study. Journal of Clinical Epidemiology 47, 1085-1093.

Samuelson G, Bratteby LE, Enghardt H \& Hedgren M (1996) Food habits and energy and nutrient intake in Swedish adolescents approaching the year 2000. Acta Paediatrica 85, Suppl. 415, 1-19.

Schofield WN, Schofield C \& James WPT (1985) Basal metabolic rate. Human Nutrition: Clinical Nutrition 39C, Suppl. 1, 1-96.

Singer MR, Moore LL, Garrahie EJ \& Ellison RC (1995) The tracking of nutrient intake in young children: the Framingham Children's Study. American Journal of Public Health 85, 1673-1677.

Stein AD, Shea S, Basch CE, Contento IR \& Zybert P (1991) Variability and tracking of nutrient intakes of preschool children based on multiple administrations of the 24-hour dietary recall. American Journal of Epidemiology 134, 14271437.

Strain JJ, Robson PJ, Livingstone MBE, Primrose ED, Savage JM, Cran GW \& Boreham CAG (1994) Estimates of food and macronutrient intake in a random sample of Northern Ireland adolescents. British Journal of Nutrition 72, 343-352.

Tanner JM (1962) In The development of the reproductive system. Growth at Adolescence, 2nd ed., pp. 28-39. Oxford: Blackwell Scientific Publications.

Twisk JWR, Kemper HCG, van Mechelen W \& Post GB (1997a) Tracking of risk factors for coronary heart disease over a 14year period: a comparison between lifestyle and biologic risk factors with data from the Amsterdam Growth and Health Study. American Journal of Epidemiology 145, 888-898.

Twisk JWR, van Mechelen W, Kemper HCG \& Post GB (1997b) The relation between long-term exposure to lifestyle during youth and young adulthood and risk factors for cardiovascular disease at adult age. Journal of Adolescent Health 20, 309-319.

van Staveren WA, de Boer JO \& Burema J (1985) Validity and reproducibility of a dietary history method estimating the usual food intake during one month. American Journal of Clinical Nutrition 42, 554-559.

Webber LS, Srinivasan SR, Wattigney WA \& Berenson GS (1991) Tracking of serum lipids and lipoproteins from childhood to adulthood. The Bogalusa Heart Study. American Journal of Epidemiology 133, 884-899.

Welten DC, Kemper HCG, Post GB, van Staveren WA \& Twisk JWR (1997) Longitudinal development and tracking of calcium and dairy intake from teenager to adult. European Journal of Clinical Nutrition 51, 612-618. 